\title{
STUDY ON CEREBRAL ANEURYSMS: RUPTURE RISK PREDICTION USING GEOMETRICAL PARAMETERS AND WALL SHEAR STRESS WITH CFD AND MACHINE LEARNING TOOLS
}

\author{
Alfredo Aranda and Alvaro Valencia \\ Department of Mechanical Engineering, \\ Universidad de Chile, Beauchef 851, Santiago 8370456, Chile.
}

\begin{abstract}
We modeled an SVM radial classification machine learning algorithm to determine the ruptured and unruptured risk of saccular cerebral aneurysms using 60 samples with 6 predictors as the gender, the age, the Womersley number, the Time-Averaged Wall Shear Stress (TAWSS), the Aspect Ratio (AR) and the bottleneck of the aneurysms, considering real cases of patients. We reconstructed computationally each geometry from an angiography image to realize a CFD simulations, where the TAWSS was computed by CFD analysis. A cross validation method was used in the training sample to validate the classification model, getting an accuracy of $92.86 \%$ in the test sample. This result may be used to help in medical decisions to avoid a complicated operation when the probability of rupture is low.
\end{abstract}

\section{KEYWORDS}

Machine Learning, CFD, cerebral aneurysm simulations, SVM, aspect ratio, computational applications.

\section{INTRODUCTION}

Millions of data are generated every day, and machine learning algorithms are used more frequently in many areas. In biology science is essential may be able to predict and reduce risk factors when a phenomenon (that may cause death[1]) is difficult to measure. An intracranial cerebral aneurysm is an abnormal dilation of an artery caused by a weakness in the wall (a reduction in the tunica media, i.e., muscular middle layer of the artery wall due to certain hemodynamic factors [2]), and it is located in the subarachnoid space at the base of the brain [3]. These are typically classified based on its form and position in the main artery into terminal, lateral, or bifurcation aneurysm [4]. High-blood pressure, smoking, family history, stress out and a previous aneurysm are main factors that may increase the risk of an aneurysm or its rupture. Clinical studies regarding the behavior of hemodynamic parameters such as wall shear stress (WSS) or the interior velocity field within the aneurysm may be occasionally difficult to measure, and indeed be able to predict whether its rupture. In contrast, a complete aneurysm hemodynamic [5] and prediction studies are possible via Computational Fluid Dynamics (CFD) reconstructing cerebral aneurysm models, and then implementing Machine Learning algorithms[6]using data generated for each simulation and the geometrical parameters. Hemodynamic stresses are considered as important factors affecting the development of aneurysms because they are directly related with aneurysm rupture risks [7][8]. Similarly, geometrical parameters (combinations 
Machine Learning and Applications: An International Journal (MLAIJ) Vol.5, No.4, December 2018

between neck, width and height of the aneurysms) may be good factors in the behavior of the aneurysm ruptures as well [9][10].

Relevant studies in machine learning techniques to predict the rupture risk of aneurysms have been developed as computing capacity increases. Judy Shum et al. [11] found in a machine learning for the assessment of AAA rupture risk study that the null hypothesis is rejected when geometrical factors are considered predictors in a classify model. Qian et al. [12] also studied the risk analysis of unruptured aneurysms using CFD and Energy Loss techniques, founding a useful tool for the prediction. An important work was developed by Bisbal et al.[13] applying a data mining approach to predict the rupture risk of aneurysms using hemodynamic, morphologic and clinical features, however, the validation of the models was not presented. Monsalve-Torra et al. [14] used a neural network algorithm to predict in hospital mortality in patients with abdominal aortic aneurysm. In addition, there are recent studies that have proposed a complete methodology to predict the risk of intracranial aneurysm ruptures using machine learning and image processing techniques, taking clinical, demographical, environmental and medical data [15]. On the other hand, a study found a first machine learning approach to establish the relationship between shape features and ascending aortic aneurysm risk predicted from FE analysis using SSM, SVM and SVR algorithms[9].Muluk et al. [16] predicted the aortic aneurysm rupture using only geometry modeling considering 15 variables. In the same way, Mocco et al. [10] used the aneurysm morphology to demonstrate and predict that these ones can be good predictors. Finally, recent studies of Liu et al. [17] and Lee et al. [18]predicted the rupture risk using morphological parameters and healthy behaviors with feed-forward artificial neural network and SVR, respectively. The objective of this investigation is carried out a supervised machine learning algorithm (SVM) using the least amount of statistic significant morphologic and hemodynamic variables, simplifying the model and the compute-time of each iteration. This work is organized as follow: Sect. 2 explain the simulation procedure and the tools to carry out all analysis. Sect. 3 presents and discusses the results. Sect. 4 draws conclusions.

\section{METHODOLOGY}

\subsection{ReConstruction Of GeOMEtries ANd Properties}

The models were generated by first performing aneurysm surgeries of the patients of Instituto de Neurocirugía Asenjo (INCA), and then reconstructing 60 three-dimensional angiography images on a 1:1 scale to obtain adequate geometries for the simulation software, ANSYS (see Figure 1). In this investigation, 30 aneurysms were previously ruptured and other 30 were previously unruptured. 
(a)

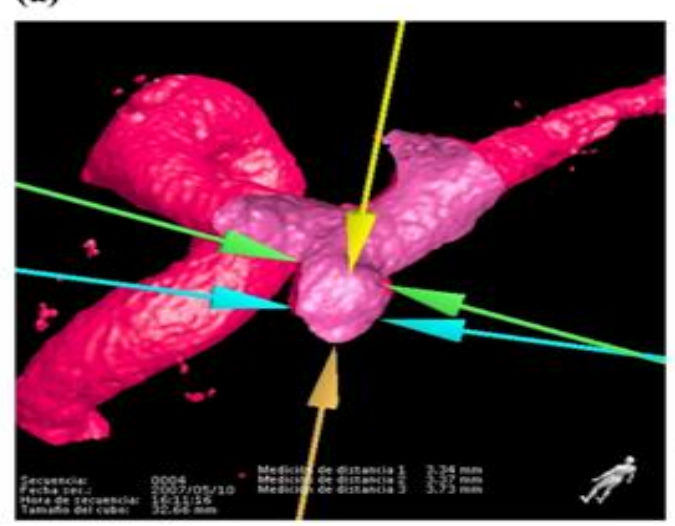

(b)

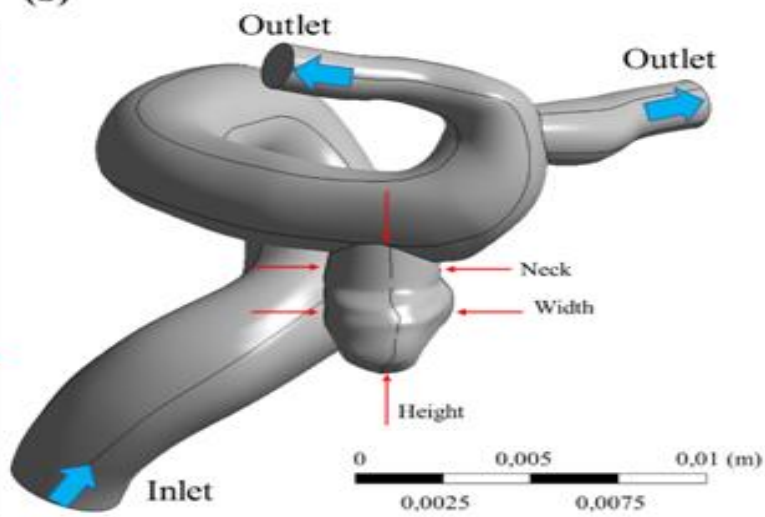

Figure 1. (a) Three-dimensional angiography image from a patient of INCA (b) Reconstruction from the three-dimensional angiography with boundary conditions (inlet and outlets) and the aneurysm geometrical measurement (Neck, Width, and Height).

The geometrical dimensions of three aneurysms are shown in Figure 1. The AR (neck-to-dome length/neck-width) was calculated for the entire cerebral aneurysm geometries, wherein AR values from 0.63 to 5.28 were considered.

A laminar and incompressible fluid flow was considered using $\rho=1065 \mathrm{~kg} / \mathrm{m}^{3}$ [19], [20] and modeled by the conservation of mass and momentum or the Navier-Stokes equations. Blood was considered as a non-Newtonian fluid, and the Carreau model was used to model the shear thinning behavior of blood, as follows [21]:

$$
\mu_{e f f}(\dot{\gamma})=\mu_{\text {inf }}+\left(\mu_{0}-\mu_{\text {inf }}\right)\left(1+(\lambda \dot{\gamma})^{2}\right)^{\frac{n-1}{2}}
$$

where $\mu_{\text {inf }}=0.00345 \mathrm{~kg} / \mathrm{m} \cdot \mathrm{s}$ (viscosity at infinite shear rate), $\mu_{0}=0.056 \mathrm{~kg} / \mathrm{m} \cdot \mathrm{s}$ (viscosity at zero shear rate), $\lambda=3.313 \mathrm{~s}$ (relaxation time), and $n=0.3568$ (power index) are the material coefficients.

Respect to the mechanic behavior of the artery walls, we considered the simplification of the rigid artery in all cases.

\subsection{Boundary Conditions}

It is important to mention that we reconstructed real aneurysms using angiography images and the boundary conditions simulated the internal cardiovascular system of the brain and these ones should be adjusted to maximize the real conditions, being an important role in the development of the flow inside each artery. While, the inlet condition was used as a velocity profile, the outlet conditions represented the blood pressure. The pressure difference was another important factor that was assumed to be only a function of time, and it was generated by a pulse wave of a finite velocity [22].

We used the Womersley velocity theory [21] to obtain the physiological flow conditions at the artery inlet. The velocity profile was computed from the general Womersley solution (Eq. 2) 
using a code in $\mathrm{C}++$ and it was subsequently exported to the ANSYS Fluent software (the Womersley solution equation is described in more detail in [21]).

$$
v(r, t)=\frac{2 Q_{0}}{\pi a^{2}}\left[1-\left(\frac{r}{a}\right)^{2}\right]+\sum_{n=1}^{N} \frac{Q_{n}}{\pi a^{2}}\left[\frac{1-\frac{J_{0}\left(\frac{\beta_{n} r}{a}\right)}{J_{0}\left(\beta_{n}\right)}}{1-\frac{2 J_{1}\left(\beta_{n}\right)}{\beta_{n} J_{0}\left(\beta_{n}\right)}}\right] e^{i n \omega t}
$$

With

$$
\beta_{n}=i^{\frac{3}{2}} \alpha_{n}=i^{\frac{3}{2}} a \sqrt{\frac{n \omega}{v}}
$$

where $\alpha_{\mathrm{n}}$ is the $\mathrm{n}$-Womersley number, a is the artery radius of the inlet, $\mathrm{n}$ is the number of modes, and $\omega$ is the angular frequency obtained from the period of the cardiac cycle. In our study, we have chosen eight modes and an angular frequency of $7.703 \mathrm{~s}-1$ (period of the cardiac cycle was $0.857 \mathrm{~s}$ ) [23]. The Womersley solution depends on the Womersley number, and it is a measure of the ratio of the momentum equation to the viscous part [21]. When $\alpha_{n}$ is small, the unsteadiness is not important, and the solutions become Poiseuille solutions that vary in magnitude, but not in shape. If $\alpha_{n}$ is large, the shapes of the profiles are not parabolic [21].

Figure 2 a shows one of 60 blood velocity profile measurements delivered by INCA. We obtained the time-averaged maximum velocity (TAMAX) following the results given by Li et al. [24] and Blanco [25] for all artery-images using an R Studio tool[26]. We localized each RGB pixel of the TAMAX line (a smooth line of the Figure 2a), then, we related the distance between a subtraction of pixels and a subtraction of centimeters at the vertical

axis. An average was derived from all the velocity profiles and it was multiplied by the inlet area of each geometry to yield the flow $\left(\mathrm{Q}_{\mathrm{i}}\right)$. Subsequently, a Fourier fitting of $\mathrm{Q}_{\mathrm{i}}$ was performed using a fit function of RStudio to obtain the respective Fourier coefficients. Finally, the Fourier coefficients were employed to calculate the Womersley velocity of each geometry.

(a)

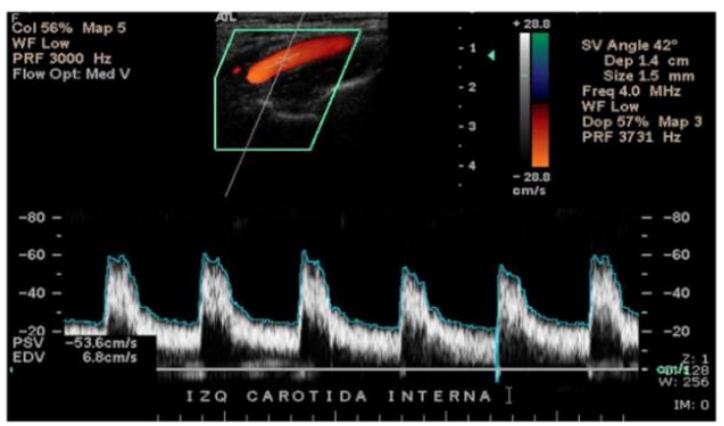

(b)

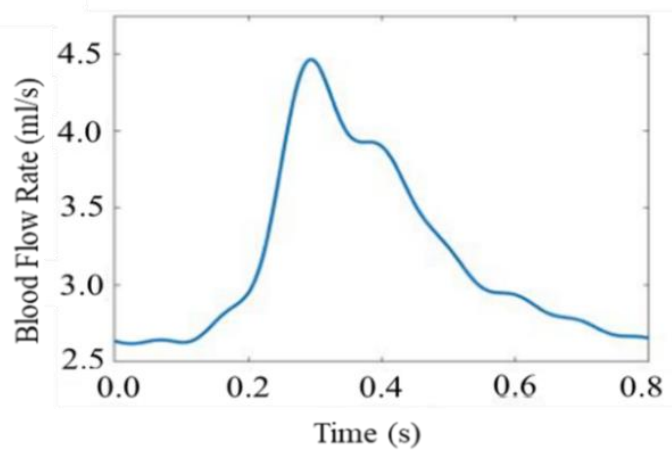

Figure 2. (a) Blood velocity measurement from a patient of INCA. (b) Flow volume to employ in the inlet condition. 
We used a normal pulsatile pressure condition between 80 and $120 \mathrm{mmHg}$ [27] (represented in Figure 3), calculated through a three-element Windkessel model for the outlets. The purpose of this model was to represent the realistic physiological flow patterns and pressure distributions in the computational domain for the fluid [28].

(a)

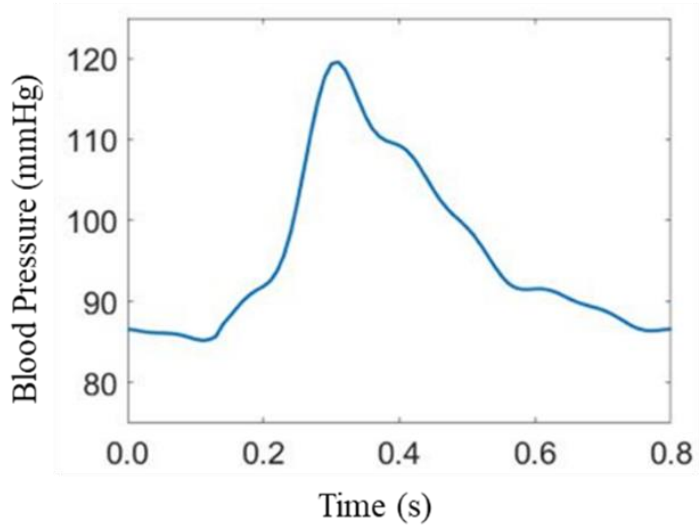

(b)

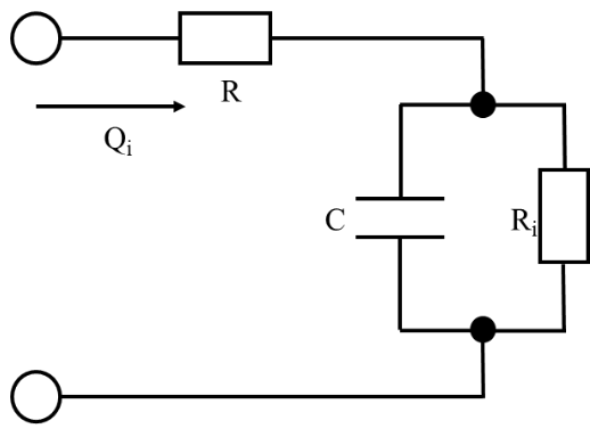

Figure 3.(a) Equivalent pulsatilepressure computed by the (b) three-element Windkessel model.

\subsection{Numerical Method and Setup of CFD}

A mesh for each geometry must be considered to solve the transient Navier-Stokes equations by considering a non-Newtonian fluid. In our case, we used a mesh of a density of 1500 elements $/ \mathrm{mm}^{3}$ (shown in Figure 4), in which we studied the difference between the mean WSS at the systole of the pulsatile blood flow with 250, 500, 1000, 2500, 3500, and 4500 elements $/ \mathrm{mm}^{3}$, respectively. The maximum difference is displayed in Figure 5, and it is below 3\% between 1500 and 4500 elements $/ \mathrm{mm}^{3}$. The criteria values selected for the time step and residual were $0.0001 \mathrm{~s}$ and 0.001 , respectively.

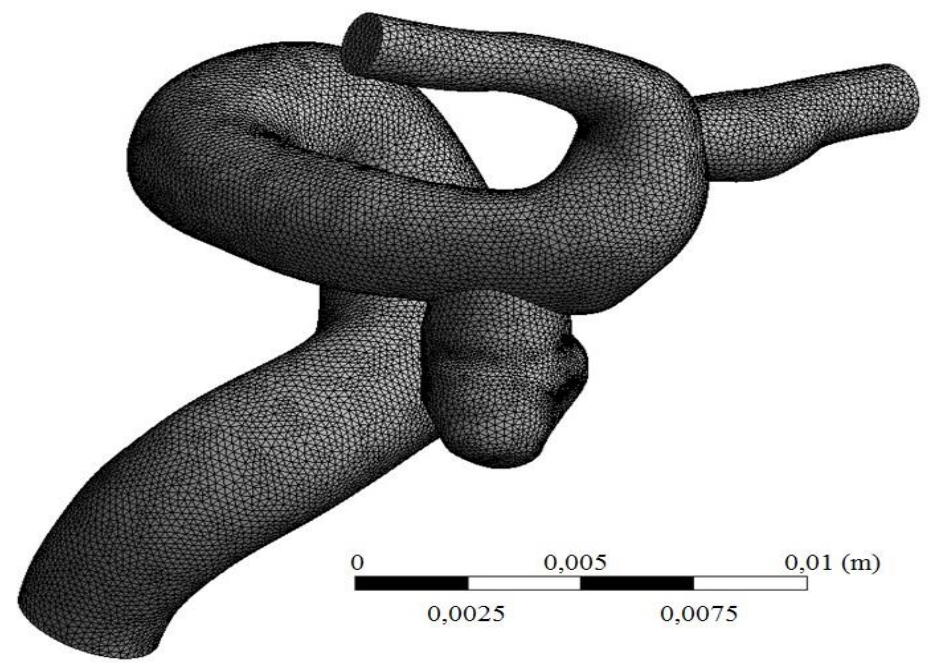

Figure 4. Type of grid using 1500 elements/mm3 for a case studied. 
Machine Learning and Applications: An International Journal (MLAIJ) Vol.5, No.4, December 2018

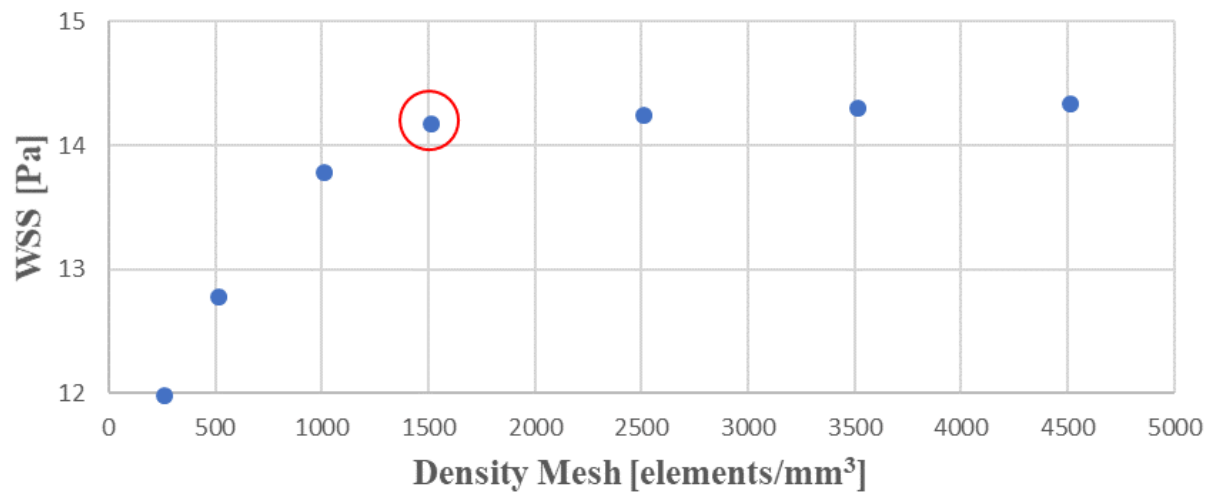

Figure 5. Comparison between the mesh results when the WSS at systole time was calculated using different mesh densities. The difference of the computing time in ANSYS was almost ten times between 4500 and 1500 elements $/ \mathrm{mm}^{3}$.

The pressure implicit with splitting of operator (PISO) algorithm was used to solve the NavierStokes equations using the spatial discretization of the least squares cell based on the gradient, second-order pressure, and second-order upwind for the momentum. For the transient formulation, the second-order implicit method was used.

We also calculated the Time-Averaged Wall Shear Stress (TAWSS) (Eq 4), where it was besides averaged over the area of each aneurysm during a cardiac cycle $\mathrm{T}$.

$$
\text { TAWSS }=\frac{1}{T} \int_{0}^{T}\left|\tau_{w}(\vec{x}, t)\right| d t
$$

\subsection{Statistic Test and Machine Learning Algorithm}

In the prediction algorithm (optimization problem) we minimized a multiclass SVM model [29] shown in Eq. (4)

$$
\min _{\theta}\left[C \sum_{k=1}^{n} \sum_{i=1}^{m} y^{(i)} \operatorname{cost}_{1}\left(\theta^{T} f^{(i, k)}\right)+\left(1-y^{(i)}\right) \operatorname{cost}_{0}\left(\theta^{T} f^{(i, k)}\right)+\frac{1}{2} \sum_{j=1}^{m} \theta_{j}^{2}\right]
$$

Where $C\left(=\frac{1}{\lambda}\right)$ is a regularization parameter to avoid the bias or variance, $y^{(i)}$ is the target, $\theta^{T}$ are the unknow variables, $x_{j}$ are the features of the model, $n$ the quantity of the predictors and $m$ quantity of the training set. We used the Gaussian kernel, $f^{(i, k)}=\exp \left(-\frac{\left\|x_{i}-x_{j}\right\|}{2 \sigma^{2}}\right)$, where features are separated for a hyperplane in the transformed space.

Also, we employed an $80 \%$ of the data for training and $20 \%$ for testing the model where an exhaustive cross-validation was used as a validation technique to avoid the problem of overfitting and it gives much more information about the algorithm performance. In addition, the features considered in the model were the gender (14 males and 46 females), the age, the Womersley number, the TAWSS, the RRT, the OSI, the AR and the Bottleneck (BNF). 
Machine Learning and Applications: An International Journal (MLAIJ) Vol.5, No.4, December 2018

Morphologic and hemodynamic parameters will be analyzed in an analysis of variance (ANOVA) test to determine the statistical significance of each one, using a tolerance smaller than 0.05 [30].

\section{RESULTS AND DISCUSSION}

\subsection{Simulation Results}

The time-dependent WSS on the wall of each cerebral aneurysm was computed in all the simulations (a case is showed in Figure 6), then, all hemodynamic parameters were computed and a SVM algorithm was developed to determine the rupture risk of each aneurysm using an RStudio script code. The WSS had a lower magnitude at the diastolic time than the systolic time, although the temporary form was maintained. In each simulation results we noted that the WSS was mainly located at the neck of the aneurysm (shown in Figure 7) due to the velocity vector inside of the cerebral artery was approximately $90^{\circ}$ with respect to the wall of a saccular aneurysm [31] forming vorticities without being significant at the dome [32][33].

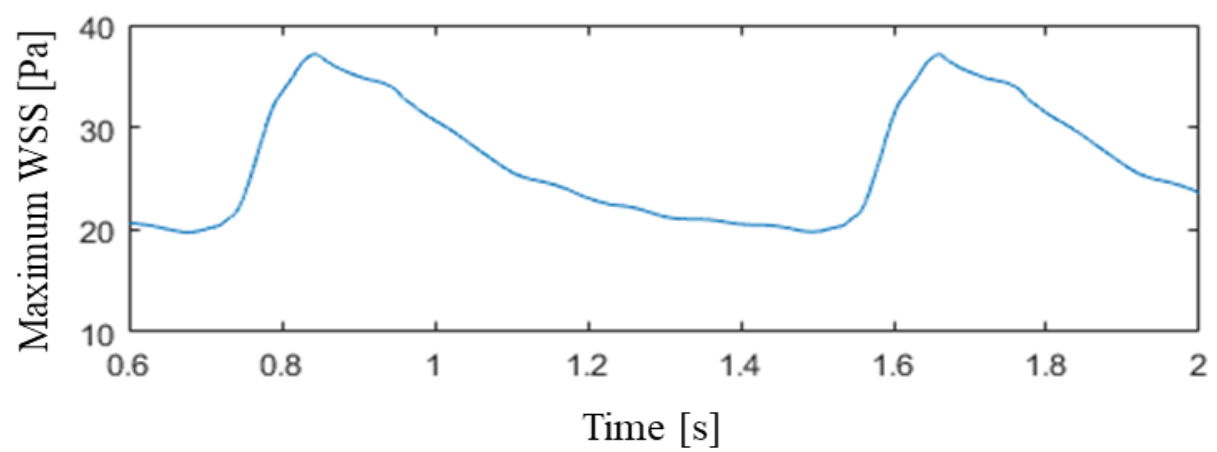

Figure 6. Mean WSS computed at an aneurysm for a normal pressure difference. We had considered the second cycle of the given pulsatile blood in each solution.
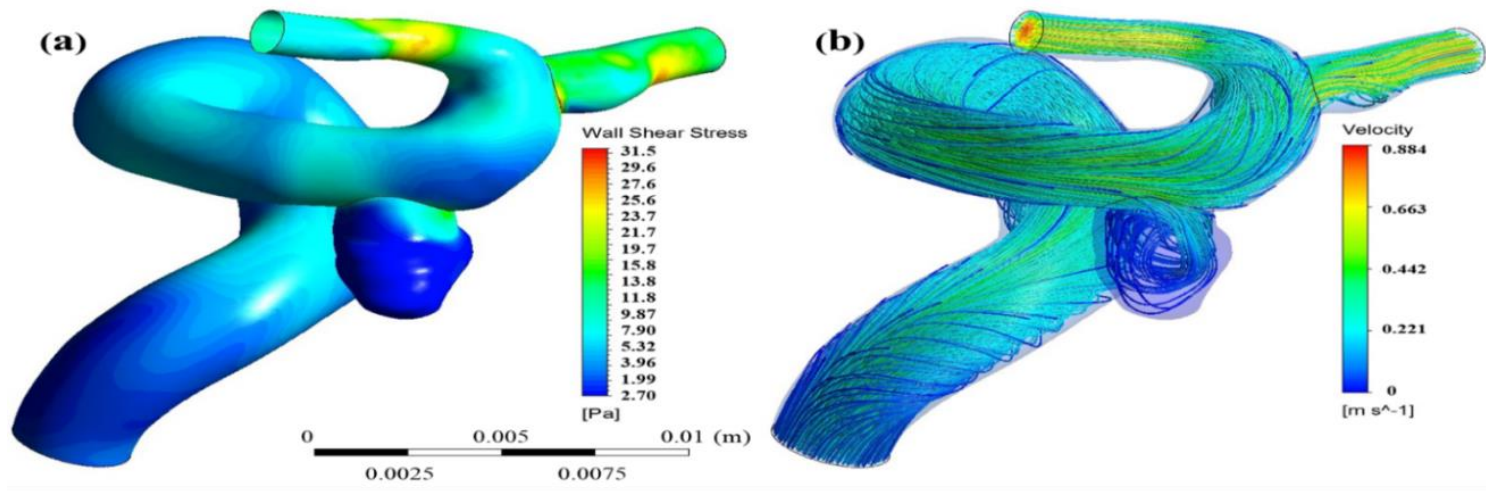

Figure 7. (a) WSS and (b) velocity streamline computed at a systolic time from ANSYS software. The WSS was localized at the neck of the aneurysm $(\mathrm{AR}=2.88)$ where the flow was facing the wall. 


\subsection{Statistical Analysis}

We analyzed the statistical significance of all parameters from CFD simulations and morphological parameters through ANOVA statistic tests, where we selected the variables shown in Table 1, satisfying the tolerance of P-value, and besides, we considered the smallest quantity of them.

Table 1. Statistic of the predictors used on the predicted models.

\begin{tabular}{ccccccc}
\hline & Statistic & WN & $\begin{array}{c}\text { TAWSS } \\
{[\mathrm{Pa}]}\end{array}$ & Age & AR & BNF \\
\hline & Min. & 0.196 & 0.253 & 29.00 & 0.854 & 0.633 \\
& 1st Qu. & 2.687 & 0.365 & 52.00 & 1.294 & 1.187 \\
Ruptured & Median & 3.303 & 0.414 & 57.00 & 1.690 & 1.453 \\
$(30$ cases $)$ & Mean & 3.004 & 0.427 & 58.67 & 1.930 & 1.819 \\
& 3rd Qu. & 3.362 & 0.523 & 67.50 & 2.303 & 2.157 \\
& Max. & 3.985 & 0.621 & 81.00 & 5.279 & 6.316 \\
\hline & Min. & 1.404 & 0.462 & 30.00 & 0.634 & 0.574 \\
& 1st Qu. & 2.423 & 0.513 & 48.25 & 1.054 & 1.012 \\
Unruptured & Median & 2.963 & 0.721 & 57.50 & 1.341 & 1.176 \\
(30 cases) & Mean & 2.871 & 0.736 & 56.10 & 1.388 & 1.372 \\
& 3rd Qu. & 3.285 & 0.828 & 64.25 & 1.551 & 1.645 \\
& Max. & 4.104 & 0.967 & 76.00 & 3.860 & 2.854 \\
\hline All cases & F Value & 5.521 & 8.361 & 5.361 & 9.968 & 4.215 \\
(60) & P Value & 0.00823 & $<0.001$ & 0.00231 & 0.00263 & 0.0095 \\
\hline
\end{tabular}

In Table 1, all quantities had higher value of the mean in the ruptured than in the unruptured group, except TAWSS, in accordance with the results obtained by Xiang et al. [34] and [35]. We also analyzed other morphological parameters such as Non-sphericity index (NSI), Aneurysm Inclination Angle (AIA), volume and area of the aneurysm being less statistically significant than $\mathrm{AR}$ and BNF, which is in good agreement with previous works [36]. Respect to the age and gender variable of the patients, the p-value is smaller than the tolerance defined, being statistically significant, following the results obtained by Li et al. [37].

\subsection{Machine Learning Results and Discussion}

An SVM with radial basis function kernel was trained with 46 samples, 6 predictors and 2 classes, being "unruptured" the positive class. To validate the model, a cross-validation methodology was implemented with 10-folds, repeated 3 times. Then, the test set was used with 14 out-of-samples. The final values used for the model were $\sigma=0.1456$ and $\mathrm{C}=0.5$, being 92.86\% in accuracy (showed in Fig. 8) and 0.6438 in Kappa. 
Machine Learning and Applications: An International Journal (MLAIJ) Vol.5, No.4, December 2018

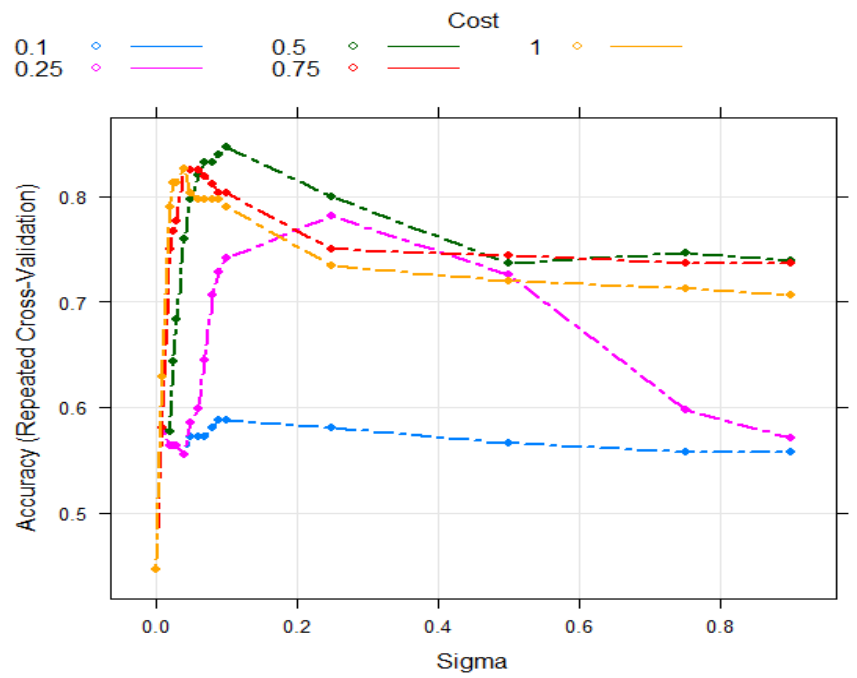

Figure 8. Combination between Sigma and Cost to compute the best accuracy to the SVM radial model.

In addition, we computed a confusion matrix for the test set where results are showed in Table 2 and the statistics parameters showed in Table 3.

Table 2. Confusion matrix for the test set using an SVM algorithm.

\begin{tabular}{ccc}
\hline Prediction & Unruptured & Ruptured \\
\hline Unruptured & 7 & 1 \\
Ruptured & 0 & 6 \\
\hline
\end{tabular}

Table 3. Statistics parameters for the validation of the model.

\begin{tabular}{cc}
\hline Statistic parameter & Value \\
\hline Accuracy & 0.9286 \\
P-Value & $<1 \mathrm{e}-4$ \\
$95 \% \mathrm{CI}$ & $(0.7613,0.9982)$ \\
Kappa & 0.8571 \\
Sensitivity & 1.0000 \\
Specificity & 0.8571 \\
Positive predictive value & 0.8750 \\
Negative predictive value & 1.0000 \\
\hline
\end{tabular}

Xiang et al. [34], Qin et al. [38], Jing et al. [39] and Fan et al. [40], used the area under the ROC curve evaluation (AUC), achieved to obtain an AUC over $70 \%$ and under $90 \%$ using morphological, biological or hemodynamic predictors. On the other hand, MonsalveTorra[14]obtained a 95.1\% in accuracy using a multilayer perceptron (MLP) algorithm, however, 57 attributes were used from 310 cases (without hemodynamic parameters), being a non-scalable problem due to high calculation times and a possible overfit of the model. Lau et al. [41] obtained a maximum accuracy of $92.03 \%$, evaluating many biological predictors in a small dataset. In this investigation, the adjusted model may predict an accuracy of $92.86 \%$ using easily measurable predictors as the AR, the Bottleneck, the age, the gender the Womersley number and the TAWSS. 
Machine Learning and Applications: An International Journal (MLAIJ) Vol.5, No.4, December 2018

Respect to the limitations of this study, computational fluid dynamic techniques in cerebral aneurysms are a relatively new approach translating a well-established engineering technology into clinical research, being a real-world approximation [42]. Boundary conditions have an important role in any CFD problem, they are approximated by no-variation generalized waveforms, quantifying hemodynamic differences between ruptured and unruptured aneurysms [43]. However, physiologic waveforms may also vary due to physical efforts or emotional excitement, resulting in a sudden change in blood pressure and TAWSS. We could also improve the accuracy of the models, considering the most statistically significant morphological, CFD, biological and fluid-structure interaction (FSI) variables, where in situations that involves the life or death of people, we need to have an accuracy as close as possible to $100 \%$. This is also possible according as we increase the number of predictors in the model, as indicated by Kohavi et al. [44], or using reinforced learning algorithms, however in both cases it would imply having more patient data, being a very difficult task to fulfill, although undoubtedly, these results may be used as support for neurosurgeons. Respect to the time-computing limitations (considering both the CFD and training simulations), we may improve it using a deep learning approach to estimate the TAWSS [45], and then, to use the results in a new model to predict the rupture risk.

\section{CONCLuSiOnS}

Six predictors were used to model an SVM radial classification algorithm to determine the ruptured and unruptured of saccular cerebral aneurysms using 60 samples. We simulated CFD models to compute the TAWSS in the aneurysms considering real cases of patients where we reconstructed each geometry from an angiography image. We assumed a rigid artery in all simulations. In the machine learning model, we considered a training sample of 48 cases randomly selected and a test sample of 12 cases. A cross validation method was used to validate the model, getting an accuracy of $92.86 \%$. This result will help in medical decisions to avoid a complicated operation when the probability of rupture is low. All predictors may be easily measurable, however, to simulate and compute the TAWSS may be slow, in consequence, to obtain a result of the ruptured or unruptured prediction in a short time, it could be investigated and modeled with a machine learning algorithm in a future investigation. Also, in a future work, fluid-structure interaction simulations may be carried out to obtain a complete scheme and outcomes from the arterial oscillation, and how some resulting force can be used such as predictor of the machine learning models.

\section{ACKNOWLEDGMENTS}

AA would like to thank CONICyT PhD fellowship No. 21140180.

\section{REFERENCES}

[1] A. Bhola and A. Kumar, "Machine Learning Based Approaches for Cancer Classification Using Gene Expression Data," Machine Learning and Applications: An International Journal, vol. 2, no. 3/4, 2015.

[2] J. Brisman, J. Song and D. Newell, "Cerebral Aneurysms," The New England Journal of Medicine, vol. 355, pp. 928-939, 2006.

[3] A. Keedy, "An overview of intracranial aneurysms," McGill Journal of Medicine, vol. 9, no. 2, pp. 141-146, 2006. 
Machine Learning and Applications: An International Journal (MLAIJ) Vol.5, No.4, December 2018

[4] M. Pritz, "Cerebral aneurysm classification based on angioarchitecture," Journal of Stroke and Cerebrovascular Diseases, vol. 20, no. 2, pp. 162-167, 2011.

[5] J. Cebral, F. Mut, J. Weir and C. Putman, "Association of hemodynamic characteristics and cerebral aneurysm rupture," American Journal of Neuroradiology, vol. 32, pp. 264-270, 2011.

[6] T. Canchi, D. Kum, E. Ng and S. Narayanan, "A Review of Computational Methods to Predict the Risk of Rupture of Abdominal Aortic Aneurysms," BioMed Research International, vol. 2015, no. 861627, p. 12, 2015.

[7] H. Steiger, "Pathophysiology of development and rupture of cerebral aneurysms," Acta Neurochirurgica Supplement, vol. 48, pp. 1-57, 1990.

[8] W. Stehbens, "Etiology of intracranial berry aneurysms," Journal of Neurosurgery, vol. 70, no. 6, pp. 823-931, 1989.

[9] L. Liang, M. Liu, C. Martin, J. Elefteriades and W. Sun, "A machine learning approach to investigate the relationship between shape features and numerically predicted risk of ascending aortic aneurysm," Biomechanics and modeling in mechanobiology, vol. 16, no. 5, pp. 1519-1533, 2017.

[10] J. Mocco, R. Brown, J. Torner, A. Capuano, K. Fargen, M. Raghavan, D. Piepgras, L. Meissner and J. Huston, "Aneurysm Morphology and Prediction of Rupture: An International Study of Unruptured Intracranial Aneurysms Analysis," Neurosurgery, vol. 82, no. 4, pp. 491-496, 2018.

[11] J. Shum, S. Elena, C. Satish and E. Finol, "Machine Learning Techniques for the Assessment of AAA Rupture Risk," in ASME 2011 Summer Bioengineering Conference, Pennsylvania, 2011.

[12] Y. Qian, H. Takao, M. Umezu and Y. Murayama, "Risk Analysis of Unruptured Aneurysms Using Computational Fluid Dynamics Technology: Preliminary Results," American Journal of Neuroradiology, vol. 32, no. 10, pp. 1948-1955, 2011.

[13] J. Bisbal, G. Engelbrecht, M. Villa-Uriol and A. Frangi, "Prediction of Cerebral Aneurysm Rupture Using Hemodynamic, Morphologic and Clinical Features: A Data Mining Approach," International Conference on Database and Expert Systems Applications, vol. 6861, pp. 59-73, 2011.

[14] A. Mosalve-Torra, D. Ruiz-Fernandez, O. Marin-Alonso, A. Soriano-Payá and J. C.-M. M. CarreñoJaimes, "Using machine learning methods for predicting inhospital mortality in patients undergoing open repair of abdominal aortic aneurysm," Journal of Biomedical Informatics, vol. 62, pp. 195-201, 2016.

[15] S. Chabert, T. Mardones, R. Riveros, M. Godoy, A. Veloz, R. Salas and P. Cox, "Applying machine learning and image feature extraction techniques to the problem of cerebral aneurysm rupture," Researchideas and Outcomes, vol. 3, p. e11731, 2017.

[16] S. Muluk, P. Muluk, J. Shium and E. Finol, "On the Use of Geometric Modeling to Predict Aortic Aneurysm Rupture," Annals of Vascular Surgery, vol. 44, pp. 190-196, 2017.

[17] J. Liu, Y. Chen, L. Lan, B. Lin, W. Chen, M. Wang, R. Li, Y. Yang, B. Zhao, Z. Hu and Y. Duan, "Prediction of rupture risk in anterior communicating artery aneurysms with a feed-forward artificial neural network," European Radiology, vol. 28, no. 8, pp. 68-75, 2018. 
Machine Learning and Applications: An International Journal (MLAIJ) Vol.5, No.4, December 2018

[18] R. Lee, D. Jarchi, R. Perera, A. Jones, I. Cassimjee, A. handa and D. Clifton, "Applied Machine Learning for the Prediction of Growth of Abdominal Aortic Aneurysm in Humans," EJVES Short Reports, vol. 39, pp. 24-28, 2018.

[19] J. Hunter, "A method of raising the specific gravity of the blood," The Journal of Physiology, vol. 11, no. 1-2, pp. 115-120, 1890.

[20] J. Hipple, Chemical Engineering for Non-Chemical Engineers, New Jersey: ALChE, 2017.

[21] M. Zamir, The Physics of Pulsatile Flow, New York: Springer, 2000.

[22] J. Womersley, "Method for the calculation of velocity, rate of flow and viscous drag in arteries when the pressure difference is known," The Journal of Physiology, vol. 127, no. 3, pp. 553-563, 1955.

[23] J. Nengom, S. Sap, D. Chelo, R. Mbono, J. Boombhi, F. Mouafo, A. Chiabi, S. kingue and P. Koki, "Assessment of cardiac function in children with congenital adrenal hyperplasia: a case control study in Cameroon," BMC Pediatrics, vol. 17, no. 109, pp. 1-8, 2017.

[24] S. Li, P. Hoskins, T. Anderson and W. McDicken, "Measurement of mean velocity during pulsatile flow using time-avegared maximum frequency of doppler ultrasound waveforms," Ultrasound in Medicine \& Biology, vol. 19, no. 2, pp. 105-113, 1993.

[25] P. Blanco, "Volumetric blood flow measurement using Doppler ultrasound: concerns about the technique," Journal of ultrasound, vol. 18, no. 2, pp. 201-204, 2015.

[26] A. Baddeley and R. Turner, "spatstat: An R Package for Analyzing Spatial Point Patterns," Journal of Statistical Software, vol. 12, no. 6, 2005.

[27] G. Ogedegbe, T. Pickering and M. Dphil, "Principles and techniques of blood pressure measurement," Cardiology Clinics, vol. 28, no. 4, pp. 571-586, 2010.

[28] N. Westerhof, F. Bosman, C. Vries and A. Noordergraaf, "Analog Studies of the Human Systemic Arterial Tre," Journal of Biomechanics, vol. 2, no. 2, pp. 121-134, 1969.

[29] L. Wang, Support Vector Machines: Theory and Applications, Springer, 2005.

[30] R. Cardinal and M. Aitken, ANOVA for the Behavioral Sciences Researcher, Psychology Press;, 2005.

[31] T. Lee, I. Borazjani and F. Sotiropoulos, "Pulsatile flow effects on the hemodynamics of intracranial aneurysm," Journal of Biomechanical Engineering, vol. 132, no. 11, pp. 1-11, 2010.

[32] C. Rossmann and J. Fisher, "Effect of Non-Newtonian Behavior on Hemodynamics of Cerebral Aneurysms," Journal of Biomechanical Engineering,, vol. 131, no. 9, 2009.

[33] V. Pereira, O. Brina, A. Gonzalez, A. Narata, P. Bijilenga, K. Schaller, K. Lovlad and R. Ouared, "Evaluation of the influence of inlet boundary conditions on computational fluid dynamics for intracranial aneurysms: A virtual experiment," Journal of Biomechanics, vol. 46, no. 9, pp. 15311539, 2013.

[34] J. Xiang, S. Natarajan, M. Tremmel, D. Ma, L. Hopkins, A. Siddiqui, E. Levy and H. Meng, "Hemodynamic-morphologic discriminants for intracranial aneurysm rupture," Stroke, vol. 42, no. 1, pp. 144-152, 2011. 
Machine Learning and Applications: An International Journal (MLAIJ) Vol.5, No.4, December 2018

[35] N. Amigo and A. Valencia, "Determining Signifcant Morphological and Hemodynamic Parameters to Assess the Rupture Risk of Cerebral Aneurysms," Journal of Medical and Biological Engineering, pp. $1-7,2018$.

[36] S. Dhar, M. Tremmel, J. Mocco, M. Minsouk, J. Yamamoto, A. Siddiqui, L. Hoplins and H. Meng, "Morphology Parameters for Intracranial Aneurysm Rupture Risk Assessment," Neurosurgery, vol. 63, no. 2, pp. 185-197, 2008.

[37] Y. Li, B. Liu, Y. Zhao, W. Yan, Y. Huang, G. Jia, D. Zhao, H. Wang, W. Zhang and J. Tian, "The risk factors of intracranial aneurysm rupture and the assessing efficacy of CTA," Biomedical Research, vol. 28, no. 13, 2017.

[38] H. Qin, Q. Yang, Q. Zhuang, J. Long, F. Yang and H. Zhang, "Morphological and hemodynamic parameters for middle cerebral artery bifurcation aneurysm rupture risk assessment.," Journal of Korean Neurosurgical Society, vol. 60, no. 5, pp. 504-510, 2017.

[39] L. Jing, J. Fang, Y. Wang, H. Li, S. Wang, X. Yang and Y. Zhang, "Morphologic and Hemodynamic Analysis in the Patients with Multiple Intracranial Aneurysms: Ruptured versus Unruptured," PlosOne, vol. 10, no. 7, 2015.

[40] J. Fang, Y. Wang, J. Liu and Y. Zhang, "Morphological-Hemodynamic Characteristics of Intracranial Bifurcation Mirror Aneurysms," World Neurosurgery, vol. 84, no. 1, 2015.

[41] Q. Lau, W. Hsu, M. Lee, Y. Mao and L. Chen, "Prediction of Cerebral Aneurysm Rupture," in 19th IEEE International Conference on Tools with Artificial Intelligence, Greece, 2008.

[42] C. Karmonik, "Toward Improving Fidelity of Computational Fluid Dynamics Simulations: Boundary Conditions Matter," American Journal of Neuroradiology, vol. 35, no. 8, pp. 1549-1550, 2014.

[43] G. Byrne, F. Mut and J. Cebral, "Quantifying the large-scale hemodynamics of intracranial aneurysms, Association of hemodynamic characteristics and cerebral aneurysm rupture, Wall shear stress on ruptured and unruptured intracranial aneurysms at the internal carotid artery," Americal Journal of Neuroradiology, vol. 35, no. 2, pp. 333-338, 2014.

[44] R. Kohavi, "A study of cross-validation and bootstrap for accuracy estimation and model selection," IJCAI, vol. 14, no. 2, pp. 1137-1145, 1995.

[45] L. Liang, M. Liu, C. Martin and W. Sun, "A deep learning approach to estimate stress distribution: a fast and accurate surrogate of finite-element analysis," Journal of the Royal Society, Interface, vol. 15, no. 138, 2018. 